Der Chemie-Preis 2015 wurde Melanie Schnell, Hamburg, in Anerkennung ihrer bahnbrechenden Entwicklungen neuer Methoden zur Untersuchung der Rotationsspektren zustandsselektierter Moleküle unter extrem kalten Bedingungen verliehen.

\title{
Melanie Schnell Untersuchungen zu Struktur, Dynamik und Chiralität polarer Moleküle mittels Rotationsspektroskopie
}

Chemische Prozesse auf der Molekülebene zu verstehen und schließlich $\mathrm{zu}$ kontrollieren ist eines der wichtigsten Ziele unserer Forschungsaktivitäten. Dafür entwickeln wir in unserer Forschungsgruppe spektroskopische Methoden, mit deren Hilfe wir komplexe Molekülsysteme und -mischungen untersuchen. Unsere Forschungsinteressen erstrecken sich von i) der Untersuchung molekularer Wechselwirkungen, die für die Molekülerkennung und damit für das Wechselspiel aus Struktur und Funktion auch von biologischen Systemen von Bedeutung sind über ii) astrochemisch-relevante Systeme hin zur iii) Entwicklung und Anwendung einer neuen Methode, mit denen sich die Enantiomere chiraler Moleküle unterscheiden lassen. Bei chiralen Mole-

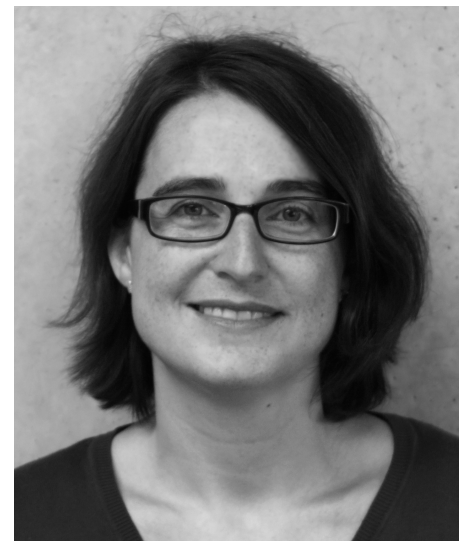

Dr. Melanie Schnell, Max-PlanckInstitut für Struktur und Dynamik der Materie Haumburg, Trägerin des Chemie-Preises 2015 külen handelt es sich um Spezies, die in zwei Formen vorliegen, die wie Spiegelbilder zueinander sind. Diese werden als Enantiomere bezeichnet. Sie haben identische physikalische Eigenschaften wie Schmelz- und Siedepunkte, können in einer chiralen, (bio)chemischen Umgebung aber komplett unterschiedliche Eigenschaften aufweisen. Dieses trifft insbesondere auch auf pharmazeutische Wirkstoffe zu, so dass heutzutage ein Großteil der neu zugelassenen Arzneimittel auf enantiomerenreinen Wirkstoffen basiert.

In diesem Preisträgervortrag werde ich mich insbesondere auf unsere Arbeiten $\mathrm{zu}$ astrochemisch relevanten Systemen und zu chiralen Molekülen konzentrieren. Auf ausführliche Literaturhinweise wird hier aufgrund des begrenzten Platzes verzichtet. Der interessierte Leser wird auf entsprechende Übersichtsartikel zu diesen Themen verwiesen, wie beispielsweise a) E. Herbst, Reviews in Modern Astronomy 19, S. 167 ff., herausgegeben von S. Röser, 2006, WILEY-VCH Verlag, Weinheim, b) 
D. Patterson, M. Schnell, Phys. Chem. Chem. Phys. 16 (2014) 11114 und c) M. Quack, J. Stohner, M. Willeke, Annu. Rev. Phys. Chem. 59 (2008) 741-769.

Die Astrochemie beschäftigt sich damit, unter welchen Bedingungen und wie sich Moleküle im Weltall bilden und weiter reagieren können. Zudem sind Moleküle hervorragende Sonden der physikalischen Bedingungen und Prozesse an ihrem jeweiligen Ort im interstellaren Raum. Bisher wurden mehr als 180 Moleküle (Stand November 2015) im Weltall identifiziert. Sie zeugen von einer reichhaltigen, komplexen Chemie. Es wurden sowohl Moleküle beobachtet, die unter terrestrischen Bedingungen stabil sind, als auch solche, die extrem reaktiv und auf der Erde nur sehr kurzlebig sind. Unter den extremen chemischen Bedingungen im interstellaren Raum (niedrige Temperaturen und geringe Dichten sowie intensive und energiereiche Strahlung) sind sie hingegen beobachtbar. Moleküle treten im interstellaren Raum unter anderem in Form riesiger Molekülwolken auf. Dichte Molekülwolken lassen kein oder nur wenig Sternenlicht durch, so dass sie für den Beobachter von der Erde aus dunkel erscheinen.

Für die Beobachtung astrochemisch relevanter Molekülsysteme und Prozesse werden u.a. Radioteleskope verwendet und die Ergebnisse mit Modellen und laborastrophysikalischen Daten verglichen. Mit Hilfe von Radioteleskopen werden die Rotationsspektren interstellarer Moleküle aufgenommen, die wie molekulare Fingerabdrücke sind und damit ihre eindeutige Zuordnung erlauben. Zurzeit gibt es grundlegende Weiterentwicklungen in diesem Bereich, wie beispielsweise die Interferometer-Teleskope. Dazu gehören das Atacama Large Millimeter/submillimeter Array (ALMA) in Chile sowie das Karl G. Jansky Very Large Array in den USA. Der interferometrische Ansatz ermöglicht es, neben den Fingerabdrücken der Moleküle über ihre Rotationsspektren auch den Ort der beobachteten Moleküle im interstellaren Raum sowie ihre Häufigkeiten sehr genau zu bestimmen. So lassen sich räumliche Karten erstellen, die die Häufigkeiten der Moleküle wiedergeben und somit interessante Einblicke in die vorgehenden chemischen Prozesse ermöglichen. Aufgrund der sehr hohen Empfindlichkeit der neuen Radioteleskope erwarten wir eine deutliche Zunahme an neuen, nicht verstandenen radioastronomischen Daten, deren Zuordnung der Unterstützung durch Labormessungen bedarf.

In unserem Labor führen wir hochauflösende spektroskopische Messungen durch, mit dem Ziel, neuartige Molekülklassen im interstellaren Raum zu entdecken sowie chemische Prozesse zu erforschen, die für ihre Entstehung und Weiterreaktion verantwortlich sind. Bisher wurden Unterschiede in Molekülhäufigkeiten im interstellaren Raum hauptsächlich auf physikalische Ursachen zurückgeführt. Heute haben wir Methoden zur Hand, auch chemische Vorgänge zu studieren. Wir führen unsere Experimente unter ähnlichen Bedingungen wie im interstellaren Raum durch. Die Molekülsysteme liegen isoliert in der Gasphase 
und bei niedrigen Dichten und Temperaturen vor. Wir beschäftigen uns unter anderem mit der Rolle polyzyklischer aromatischer Kohlenwasserstoffe (polycyclic aromatic hydrocarbons, PAHs) im interstellaren Raum. Es wird vermutet, dass sie für die unidentifizierten Infrarotbanden und die diffusen interstellaren Banden verantwortlich sind, die bereits vor einigen Jahrzehnten erstmals vermessen wurden. Ihr (molekularer) Ursprung ist allerdings noch weitgehend unbekannt. Zudem wird geschätzt, dass 10-20\% des gesamten galaktischen Kohlenstoffs in PAHs gebunden sind. Interessante offene Fragestellungen in diesem Zusammenhang beziehen sich zum einen auf die Strukturen dieser Moleküle, wobei es hier auch ein Ziel ist, quantenchemische Methoden zu validieren und gute Startstrukturen für weiterführende Experimente zu liefern. Darüber hinaus ist es interessant herauszufinden, welche Rolle PAHs in der noch weitgehend unverstandenen interstellaren Chemie spielen, z. B. als Katalysatoren.

Ein Schwerpunkt unserer experimentellen Methoden liegt auf der hochauflösenden Rotationsspektroskopie. Strahlung verschiedener Wellenlänge hat unterschiedliche Auswirkungen auf Moleküle. Kurzwellige, ultraviolette Strahlung beispielsweise führt zu elektronischen Anregungen in Molekülen. Langwelligere Infrarotstrahlung regt sie zu Schwingungen an, während die noch langwelligere Mikrowellenstrahlung Moleküle zur Rotation anregt. Da Moleküle Quantensysteme sind, erfolgen diese Anregungen nicht kontinuierlich, sondern diskret bei bestimmten Wellenlängen und nach besonderen Regeln. Das führt dazu, dass hochaufgelöste Molekülspektren für verschiedene Spezies sehr charakteristisch sein können und eine eindeutige Zuordnung erlauben. Da die Rotationsbewegung eines Körpers, hier eines Moleküls, direkt von seiner Struktur abhängt, lassen sich Molekülstrukturen sehr genau bestimmen.

Abbildung 1 zeigt das Breitband-Rotationsspektrum des Acenapthens, einem PAH bestehend aus drei Kohlenstoffringen, sowie spektrale Ausschnitte für Komplexe des Acenapthens mit einem, zwei und drei Wassermolekülen. Diese Wasserkomplexe sind nicht nur aus astrochemischer Sicht für ein besseres Verständnis der Bildung von sogenannten Eis-Staub-Partikeln von Interesse, sondern auch für die Aerosolbildung, da PAHs auch eine wichtige Rolle als Verbrennungsprodukte einnehmen.

Aus den Strukturen der Komplexe wird die Dominanz der Wasser-WasserWechselwirkungen ersichtlich. Die Wassermoleküle scheinen miteinander Cluster zu bilden, die dann mit den ausgedehnten Elektronensystemen der PAHs wechselwirken. D. h. sie verwenden die PAHs als Plattform. Mit Hilfe der Isotopensubstitution in natürlicher Häufigkeit, wie im Fall des ${ }^{13} \mathrm{C}$-Isotops mit ca. 1\%iger natürlicher Häufigkeit, oder unter Verwendung isotopen-angereicherter Proben wie dem Wasser-Isotopologen $\mathrm{H}_{2}{ }^{18} \mathrm{O}$ lassen sich Molekülstrukturen präzise und eindeutig bestimmen. 
(a)

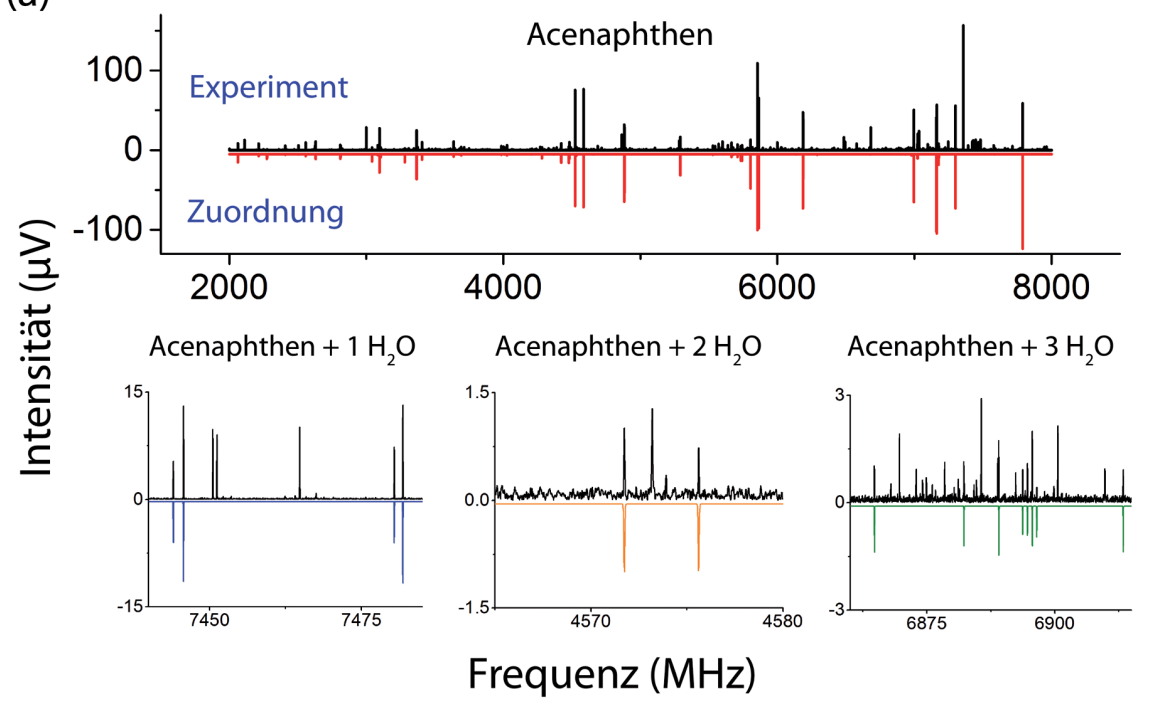

(b)

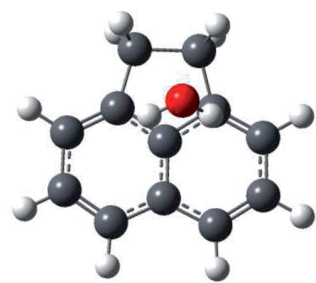

Acenaphthen $+1 \mathrm{H}_{2} \mathrm{O}$

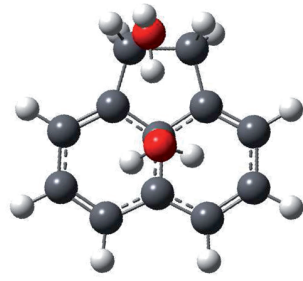

Acenaphthen $+2 \mathrm{H}_{2} \mathrm{O}$

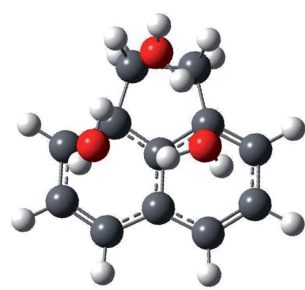

Acenaphthen $+3 \mathrm{H}_{2} \mathrm{O}$

Abb. 1: Breitband-Rotationsspektrum im Frequenzbereich von 2000-8000 MHz des polyzyklischen aromatischen Kohlenwasserstoffs Acenapthen sowie seiner Komplexe mit einem, zwei und drei Wassermolekülen (Teil (a)). In Teil (b) sind berechnete Strukturen (MP2/ aug-ccpVTZ) der Acenaphthen-Wasserkomplexe dargestellt. Sie verdeutlichen die dominierenden Wasser-Wasser-Wechselwirkungen.

Die Wassermoleküle in diesen Systemen zeigen eine komplexe Dynamik, d.h. die Wassermoleküle stehen bezogen auf den Rest des Moleküls nicht still, sondern führen interne Drehungen durch. Diese werden von uns zurzeit im Detail untersucht. In der nahen Zukunft werden wir zum einen unsere Arbeiten auf verwandte PAH-Klassen wie stickstoffsubstituierte Spezies erweitern, um ihre Moleküleigenschaften genau zu bestimmen und für die Radioastronomie zugänglich zu machen. Zum anderen werden wir uns verstärkt mit ihren Wasserkomplexen mit zunehmender Anzahl an Wassermolekülen beschäftigen. Der Nachweis derartiger größerer Komplexe im interstellaren Raum kann 
hilfreichen Aufschluss über die Ausbildung komplexer Molekülstrukturen geben.

In einer anderen Forschungsrichtung, die ich Ihnen in diesem Preisträgervortrag näher vorstellen möchte, entwickeln wir neue Methoden, mit denen sich komplexe Proben chiraler Moleküle analysieren lassen. Chirale Moleküle sind in unserem Leben allgegenwärtig. Zucker, Aminsosäuren sowie eine zunehmende Anzahl pharmazeutischer Wirkstoffe sind chiral. Wie bereits eingangs erwähnt handelt es sich dabei um Spezies, die in zwei Formen vorliegen, die wie Spiegelbilder zueinander sind und als Enantiomere bezeichnet werden. Diese Enantiomere haben identische physikalische Eigenschaften wie Schmelz- und Siedepunkte, können in einer chiralen, (bio)chemischen Umgebung aber vollkommen verschiedene Eigenschaften haben, worauf ihre große pharmazeutische Bedeutung basiert. Beispielsweise weist nur ein Enantiomer des Ibuprofens die bekannten entzündungshemmenden und schmerzlindernden Eigenschaften auf, während das andere Enantiomer in unserem Körper keinen Effekt hat. Ein anderes Beispiel für die unterschiedlichen Eigenschaften chiraler Moleküle in einer biochemischen Umgebung ist mit unserem Geruchssinn verknüpft. Während das eine Enantiomer des Carvon-Moleküls nach Minze riecht, verbinden wir den Geruch seines Spiegelbilds mit Kümmel. Aufgrund ihrer weiten Verbreitung und großen Bedeutung ist es daher nicht überraschend, dass in den letzten 160 Jahren seit Entdeckung der Chiralität durch Louis Pasteur im Jahre 1848 zahlreiche Methoden entwickelt wurden, mit denen sich chirale Moleküle analysieren, die beiden Enantiomere unterscheiden und einer bestimmten Händigkeit zuordnen lassen. Allerdings beruhen einige dieser Methoden auf nur schwachen Effekten, manche wiederum sind nur begrenzt mischungskompatibel (insbesondere wenn mehrere chirale Moleküle in einer Mischung vorliegen) oder sie setzen eine aufwendige Probenvorbereitung voraus.

Wir haben kürzlich zusammen mit Prof. John Doyle und Dr. David Patterson von der Harvard Universität eine neue Methode zur Untersuchung chiraler Moleküle entwickelt, die auf der Mikrowellenspektroskopie basiert und damit auch ihre hochauflösenden und spezies-sensitiven Eigenschaften aufweist. Die Methode ist daher inhärent mischungskompatibel. Sie basiert auf dem Spiegelbildcharakter der beiden Enantiomere, der auch auf ihre Dipolmomente zutrifft (Abbildung 2). Das Dipolmoment eines Moleküls resultiert aus einer räumlichen Ladungstrennung innerhalb des Moleküls (obwohl es wie in den hier diskutierten Fällen nach außen hin neutral sein kann). Ein Beispiel ist das Wassermolekül, $\mathrm{H}_{2} \mathrm{O}$. Das Sauerstoffatom $\mathrm{O}$ ist elektronegativer als die beiden Wasserstoffatome $\mathrm{H}$, d.h. es hat eine höhere Neigung, Elektronen anzuziehen. Daher liegt am Sauerstoffatom des $\mathrm{H}_{2} \mathrm{O}$-Moleküls eine höhere Elektronendichte vor als bei den Wasserstoffatomen, d.h. eine negative Partialladung. Daraus resultiert eine ungleiche 
Elektronenverteilung im Molekül. Man spricht auch von polaren Molekülen. Da die Dipolmomente eines Moleküls direkt mit ihrer Struktur verknüpft sind, verhalten sich auch diese für die beiden Enantiomere chiraler Moleküle wie Bild und Spiegelbild (Abbildung 2).

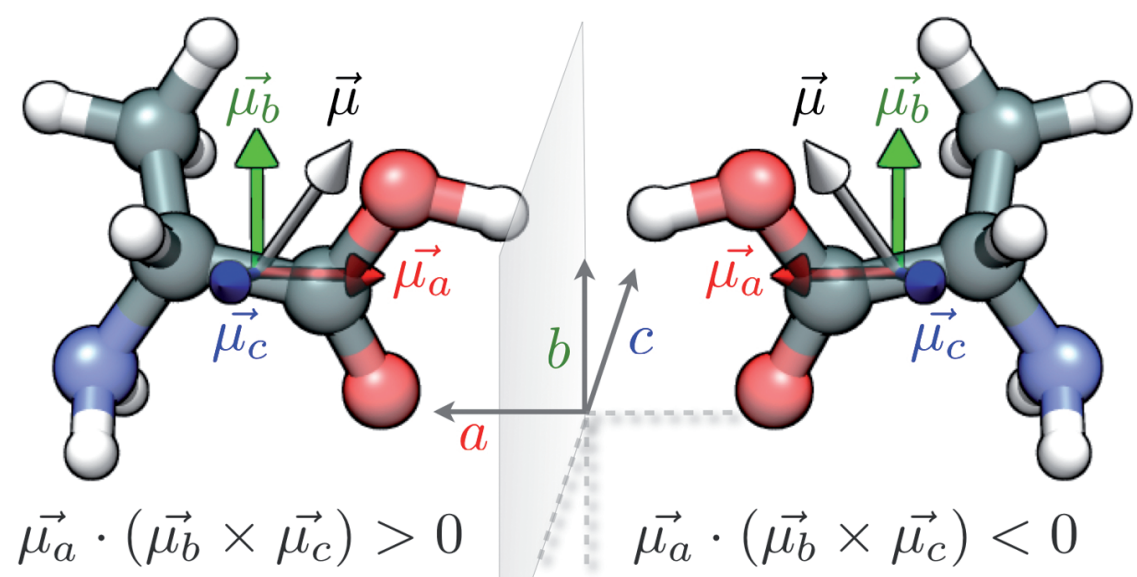

Abb. 2: Spiegelbildcharakter der Enantiomere und ihrer Dipolmomente, hier verdeutlicht am Beispiel der Aminosäure Alanin. Dieser Spiegelbildcharakter führt zu entgegengesetzten Vorzeichen des Produkts der Dipolmomentskomponenten $\mu_{\mathrm{a}}, \mu_{\mathrm{b}}$ und $\mu_{\mathrm{c}}$. Es handelt sich dabei um die Dipolmomentskomponenten bezogen auf die Hauptträgheitsachsen a, b und c des Moleküls. In unseren chiralitätssensitiven Experimenten führt dieser Vorzeichenwechsel zu einem charakteristischen Phasenunterschied für die beiden Enantiomere.

Diesen Spiegelbildcharakter können wir in unseren neuartigen Experimenten ausnutzen, indem wir eine Kombination maßgeschneiderter Mikrowellenpulse in einer Art Doppelresonanzexperiment verwenden, in dem die Polarisationsrichtungen der beiden Anregungspulse orthogonal zueinander sind. Wir erhalten dann eine Molekülantwort in der verbleibenden dritten, orthogonalen Polarisationsrichtung, die für die beiden Enantiomere verschieden und somit chiralitätssensitiv ist (Abbildung 3).

Da drei verschiedene Mikrowellenpulse in dem Schema involviert sind, bezeichnen wir es auch als Mikrowellen-Drei-Wellen-Mischen. Zudem werden die Moleküle wie in der normalen Mikrowellenspektroskopie resonant angeregt, so dass diese Methode auch auf Mischungen chiraler Moleküle anwendbar ist. Ein Beispiel ist die Untersuchung der Inhaltsstoffe essentieller Öle wie dem Pfefferminzöl, welches hauptsächlich aus verschiedenen chiralen Molekülen besteht. 
(a)

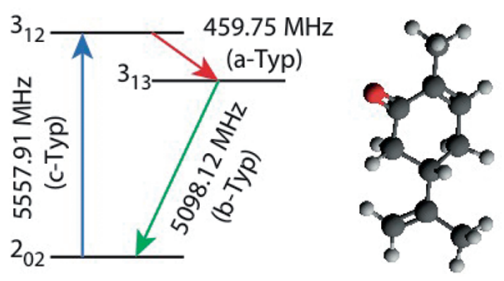

(b)

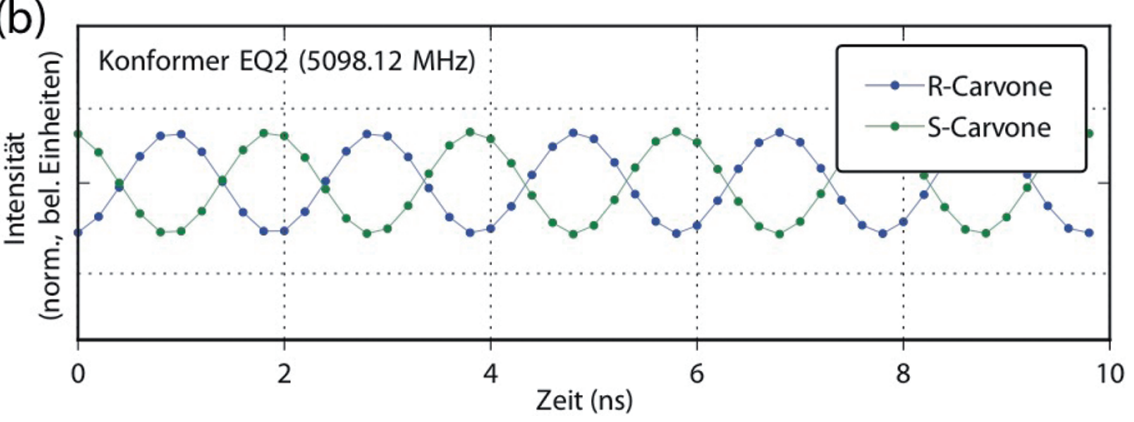

Abb. 3: Drei-Wellen-Mischen zur Unterscheidung der beiden Enantiomere des Carvons. In Teil (a) sind die verwendeten Zyklen aus drei Rotationsübergängen, die jeweils die Dipolmomentskomponenten $\mu_{\mathrm{a}}, \mu_{\mathrm{b}}$ und $\mu_{\mathrm{c}}$ involvieren, dargestellt. Im unteren Teil (b) sind die gemessenen Phasenunterschiede für die beiden Enantiomere (grün und blau) im sogenannten Freien Induktionszerfall (FID) gezeigt.

Da es sich um ein pflanzliches Öl natürlichen Ursprungs handelt, liegen die chiralen Moleküle üblicherweise nicht als Razemat vor, d.h. beide Enantiomere sind nicht zu gleichen Teilen enthalten, sondern mit einem natürlichen Enantiomerenüberschuss. Sieben verschiedene Terpen-Moleküle konnten eindeutig mit Hilfe ihrer Rotationsspektren nachgewiesen werden (Abbildung 4).

Die neue Methode des Mikrowellen-Drei-Wellen-Mischens ist also besonders interessant für eine zukünftige Erweiterung auf Proben biologischer oder pharmazeutischer Bedeutung. Darüber hinaus arbeiten wir zurzeit an Experimenten, mit denen sich die Zusammensetzung chiraler Proben beeinflussen und schließlich kontrollieren lässt. Die Verwendung wohl definierter, resonanter Mikrowellenpulse kann hier eine neue Richtung faszinierender Molekülphysikexperimente eröffnen. 
(a)

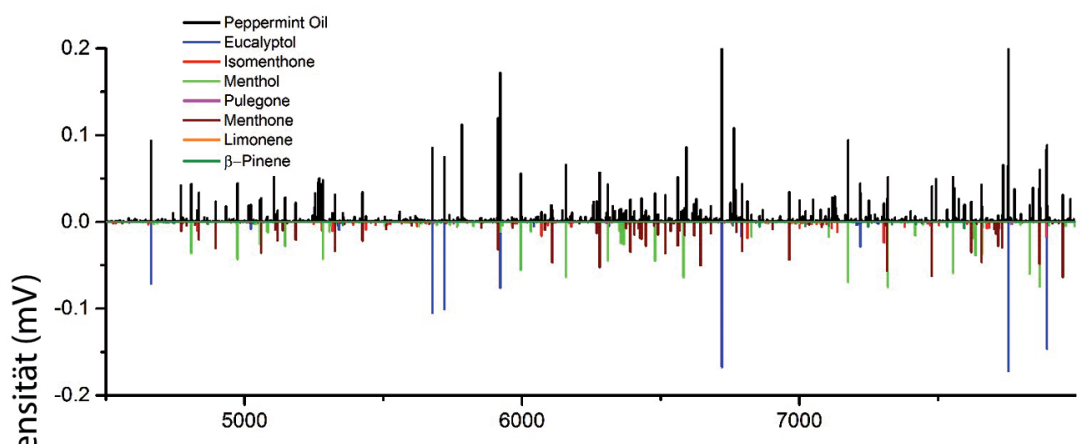

(b)

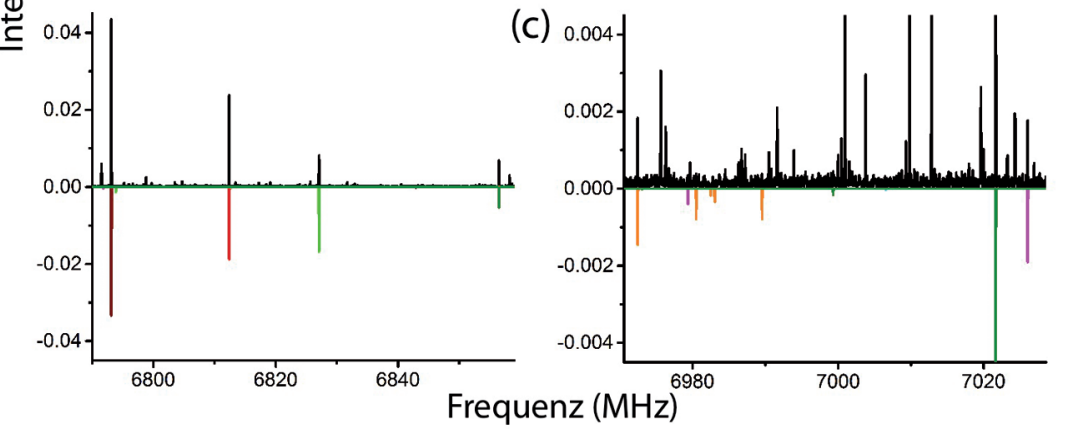

Abb. 4: Ausschnitt aus dem Breitband-Rotationsspektrum des kommerziell erhältlichen Pfefferminzöls. Teil (a) zeigt einen Breitband-Bereich von 4500-8000 MHz, während Teil (b) und (c) ausgesuchte Bereiche darstellen, die verdeutlichen, dass die verschiedenen Moleküle eindeutig mit Hilfe ihrer Rotationsspektren unterschieden werden können. Es ist zu beachten, dass sich die Intensitätsskalen der drei Darstellungen um jeweils eine Größenordnung unterscheiden. 